\title{
Estudio comparativo de la centralidad y uso de Twitter de las televisiones autonómicas en los debates electorales del $26 \mathrm{M}$
}

\section{Comparative study of the centrality and use of Twitter of regional televisions in the electoral debates of M-26}

Belén Galletero Campos. Universidad de Castilla-La Mancha. España.

belen.galletero@uclm.es

$[\underline{\mathrm{CV}}]$ (1)

Ana María López Cepeda. Universidad de Castilla-La Mancha. España.

ana.lopezcepeda@uclm.es

$[\underline{\mathrm{CV}}]$ (1) $\mathrm{G}$

Arturo Martínez Rodrigo. Universidad de Castilla-La Mancha. España.

arturo.martinez@uclm.es

$[\underline{\mathrm{CV}}] \odot \mathrm{C}$

Este artículo forma parte de los trabajos desarrollados en el marco del proyecto de investigación (RTI2018096065-B-I00) del Programa Estatal de I+D+I orientado a los Retos de la Sociedad del Ministerio de Ciencia, Innovación y Universidades (MCIU), Agencia Estatal de Investigación (AEI) y del Fondo Europeo de Desarrollo Regional (FEDER) sobre "Nuevos valores, gobernanza, financiación y servicios audiovisuales públicos para la sociedad de Internet: contrastes europeos y españoles".

Cómo citar este artículo / Referencia normalizada

Galletero Campos, B., López Cepeda, A. M. y Martínez Rodrigo, A. (2020). Estudio comparativo de la centralidad y uso de Twitter de las televisiones autonómicas en los debates electorales del 26 M. Revista Latina de Comunicación Social, (76), 97-119. https://www.doi.org/10.4185/RLCS2020-1439

\begin{abstract}
RESUMEN
Introducción: Los debates electorales televisivos se ajustan a unas condiciones previamente pactadas, dentro de los cánones legales establecidos por la LOREG. Sin embargo, en el actual entorno comunicativo se produce un debate paralelo en la segunda pantalla sujeto a menor control. En este trabajo se analiza la conversación generada en Twitter durante ocho debates emitidos por las televisiones autonómicas durante la campaña del 26 de mayo de 2019. Metodología: Mediante el hashtag proporcionado para cada uno de ellos, se ha realizado un análisis de los principales núcleos de interacción y de la actividad de los perfiles oficiales de las televisiones durante los encuentros. Resultados y conclusiones: Los resultados indican que sólo en algunos casos las televisiones logran ser relevantes en el debate paralelo en Twitter y que apenas utilizan el potencial de la red social para generar conversación. Por otro lado, la equidad a la hora de mencionar a partidos políticos y candidatos queda supeditada a la propia deontología de cada ente.
\end{abstract}


PALABRAS CLAVE: debates electorales; Twitter; televisión; comunidades autónomas; centralidad.

\begin{abstract}
Introduction: The electoral debates on television are consistent with previously agreed conditions, within the legal canons established by the LOREG. However, in the current communicative environment, there exists a parallel debate on the second screen which is not subjected to any control. This paper analyzes the conversation generated on Twitter during eight debates, broadcasted by the regional television corporations during the campaign of May 26, 2019. Methodology: Using the hashtag provided for each TV entity, an analysis of the main nodes interaction and the activity of the official Twitter profiles of televisions during the meetings has been performed. Results and conclusions: The results indicate that only in some cases televisions manage to be relevant in the parallel debate on Twitter and that they hardly use the potential of the social network to generate conversation. On the other hand, equity from TV corporations is subjected to their own deontology, when they mention to different political parties on Twitter.
\end{abstract}

KEYWORDS: electoral debates; Twitter; television; regions; centrality.

\title{
CONTENIDOS
}

1. Introducción. 1.1. Objetivos. 2. Regulación de la información en campaña electoral. 2.1. Campaña electoral y redes sociales. 3. Método. 3.1. Características de la muestra. 3.2. Procedimiento para la extracción de tuits y formación de los sociogramas. 3.3. Cálculo de métricas de centralidad 3.3.1. Grado de centralidad (Degree centrality). 3.3.2. Centralidad de intermediación (Betweeness centrality). 3.3.3. Centralidad propia (Eigencentrality). 4. Resultados. 4.1. Audiencia tradicional, conversación en Twitter y evolución temporal del hashtag. 4.2. Comportamiento del nodo y medidas de centralidad. 4.2.1. Televisiones centrales: Telemadrid, Castilla-La Mancha Media y La 7 Castilla y León. 4.2.2. El caso intermedio: Aragón TV y Canal Extremadura. 4.2.3. Televisiones sin actividad relevante: Televisión Canaria, La 7 (Murcia) y TPA (Asturias). 5. Discusión y conclusiones. 6. Referencias bibliográficas.

\section{Introducción}

La concurrencia de varias citas electorales durante 2019 ha mantenido el interés por la información política. En este contexto, la pertinencia de realizar debates electorales ha ganado peso, favorecida por la aparición de mayor número de opciones políticas $\mathrm{y}$, por tanto, un escenario de mayor competencia para transmitir los mensajes electorales. Los primeros debates electorales en España tuvieron lugar en 1993 pero ha sido en las últimas convocatorias cuando se han consolidado como un acto más dentro de la campaña si hablamos de las elecciones generales, no así en convocatorias autonómicas. En mayo de 2019 varias televisiones regionales han estrenado este formato, lo que plantea por primera vez la oportunidad de realizar un análisis comparativo no circunscrito a las grandes cadenas nacionales. Factores como la espectacularización de la política (Padilla-Castillo, 2015) y la aparición de un escenario más competitivo con nuevas formaciones concurrentes, han favorecido que las televisiones apuesten por este formato y los datos confirman que el público ha respondido. Los dos debates electorales de ámbito nacional entre los líderes de las principales formaciones -el de TVE emitido el 22 de abril y el de Atresmedia emitido al día siguienteencabezan el ranking de las emisiones más visitas durante el mes de abril, con un 38,5\% y un 27,2\% de share respectivamente ${ }^{1}$, sin tener en cuenta la audiencia acumulada en las emisiones simultáneas en otros canales. En las televisiones autonómicas las audiencias alcanzadas van desde los resultados

${ }^{1}$ Datos extraídos del informe mensual de audiencias de la consultora Barlovento Comunicación 
más modestos de la televisión de Murcia, con un 2,5\% de cuota de pantalla, hasta el 8-9\% registrado por la televisión castellanomanchega, la de Asturias o Telemadrid ${ }^{2}$.

La televisión actual se enfrenta al reto de mantener la atención de la audiencia en los contenidos televisivos, pero, al mismo tiempo, trasladarla a las redes sociales, de tal manera que se articulen comunidades que comenten aquellos contenidos vistos (Gómez Aguilar, Paniagua Rojano y Farias Batlle, 2015). Se trata de intentar rentabilizar el esfuerzo en la producción de estos espacios maximizando su alcance, algo que se puede lograr con mensajes de autopromoción previos a la emisión del debate pero también liderando la conversación en otras plataformas.

De acuerdo a la definición de Harboe $(2009$, p. 7), televisión social es aquella que "usa la tecnología de la comunicación para conectar a los telespectadores, con el fin de crear experiencias compartidas a distancia en torno a los contenidos televisivos". Por tanto, la aparición de las redes sociales ha propiciado que al tradicional número o porcentaje de espectadores de un programa se una la audiencia social, aquella que participa activamente en la experiencia de visionado mediante la interacción en una segunda pantalla (Lochrie y Coulton, 2012; Giglietto y Selva, 2014) - sea horizontal con otros miembros del público, sea vertical con el emisor - de manera simultánea a la emisión (Iñesta Fernández, 2015). Algunas motivaciones de los usuarios para establecer estas interacciones son comunicar sus impresiones sobre una emisión, buscar y compartir información, experimentar la sensación de un visionado compartido, sentir curiosidad sobre la opinión de los otros y recomendar programas (Han y Lee, 2014).

En este sentido, Twitter constituye una plataforma idónea para amplificar el alcance de la emisión televisiva, al ser una red de acceso público que favorece la transmisión viral de la información (Sequera Díaz, 2013). Además, el uso de hashtags o etiquetas evita la dispersión y favorece una conversación estructurada. La pregunta es: ¿quién domina esa conversación durante la emisión de estos espacios? Las metodologías e indicadores para determinar la influencia de perfiles y hashtags son variadas. Una de las herramientas más utilizadas en el pasado para medir la popularidad en esta red fue el factor Klout, obtenido a partir de seguidores, grado de conversación e influencia de los seguidores (Serrano-Puche, 2012). Sin embargo, la empresa que proporcionaba esta métrica a partir de sus propios algoritmos suspendió el servicio en mayo de 2018.

Desde 2013 se localiza cierta producción científica orientada a analizar, mediante estudios de caso, el comportamiento de la llamada audiencia social, aunque los trabajos suelen estar centrados en formatos de entretenimiento, ya sea en prime time (Saavedra Llamas, Rodríguez Fernández y Barón Dulce, 2015; Rodríguez-Vázquez, Direito-Rebollal y Lago-Vázquez, 2016), talent shows (QuintasFroufe y González-Neira, 2014) retransmisiones en directo (Congosto, Deltell, Claes y Osteso López, 2013a) o ficción (Deltell Escolar, Claes y Osteso López, 2013). Una conclusión común a estos estudios es la relación entre el contenido emocional y/o polémico y una mayor participación de este tipo de audiencia. La comunicación política, sin embargo, no se encuentra muy alejada de este componente de emociones y sentimientos, como han señalado algunos autores (Rúas- Araújo, Puentes-Rivera y Dapena-González, 2015), tanto en lo que se refiere a discursos de los representantes políticos como en la decisión de voto. Así, la capacidad predictiva de Twitter en el comportamiento electoral ha sido puesta a prueba en numerosos estudios del ámbito nacional (Congosto y Aragón, 2012) e internacional (Tumasjan, Sprenger, Sandner y Welpe, 2010; Asur y Huberman, 2010; Larsson y Moe, 2011; Tjong y Bos, 2012).

${ }^{2}$ Datos difundidos en nota de prensa por Castilla-La Mancha Media 
Más recientes son las aportaciones de Lago Vázquez, Direito Rebollal, Rodríguez Vázquez y López García (2016) sobre el consumo y seguimiento de la campaña electoral en las elecciones generales de 2015 por parte de la generación millenial, basada en un enfoque cualitativo, y la de Vergeer y Hans Franses (2015), que aplican análisis del contenido a la retransmisión y a los tuits. Como concluye Campos-Domínguez (2017), los análisis del debate generado en Twitter, y, en particular, el mapeo de conversaciones políticas, se mantienen en los últimos años como foco de estudio en el entorno científico internacional. Sin embargo, no han sido aplicados durante la emisión de debates políticos de carácter autonómicos. Este estudio comparado entre los ocho debates permitirá detectar diferencias en el impacto que éstos tuvieron en la red social.

\subsection{Objetivos}

El objetivo principal de esta investigación es analizar qué papel ocuparon las televisiones autonómicas en la conversación que se generó en Twitter durante los debates electorales autonómicos, celebrados en mayo de 2019.

Enmarcados en este propósito, se detallan los siguientes objetivos secundarios:

- Comparar el alcance del hashtag en proporción al número de seguidores de cada perfil corporativo y en proporción a la audiencia televisiva alcanzada en el debate.

- Comprobar cuáles fueron los actores más relevantes en el caso de los hashtags que más conversación generaron.

- Analizar y comparar la importancia, en términos de centralidad, del perfil de Twitter de cada televisión.

- Comparar las dinámicas de emisión y difusión de mensajes en Twitter por parte de las televisiones autonómicas, a partir de la medición de menciones recibidas y menciones realizadas.

- Comprobar la evolución temporal de la conversación en torno a la etiqueta del debate.

\section{Regulación de la información en campaña electoral}

La información y la comunicación persuasiva en campaña electoral está sujeta a una serie de normativas que delimitan el papel de los medios de comunicación, en aras de mantener la neutralidad y la equidad de trato a todas las formaciones políticas. En la regulación sobre información en campañas electorales, recogida en la LOREG (Ley Orgánica 5/1985, de 19 de junio, del Régimen Electoral General), se pueden diferenciar cuatro niveles de control según el medio de comunicación de que se trate: a. Un nivel máximo, aplicable a la radiotelevisión pública, a la que se le exige el respeto al pluralismo político y social, a la igualdad, proporcionalidad y neutralidad informativa; $b$. Un nivel intermedio, referido a la televisión privada, medio en el que se deberán respetar los principios de pluralismo e igualdad en todo caso y los principios de proporcionalidad y neutralidad informativa únicamente en los debates, entrevistas e información electoral; c) Un nivel atenuado, que rige para las emisoras de titularidad privada, que solo deberán respetar los principios de igualdad y pluralismo; d) Un nivel mínimo para la prensa escrita, únicamente sujeta al derecho de rectificación (Holgado González, 2017, pp. 469-470).

En cierto modo, se puede afirmar que esta regulación se ha quedado obsoleta por no contemplar apenas cuestiones como el tratamiento informativo de medios digitales o herramientas como las redes sociales, que ya no pueden considerarse un fenómeno incipiente. La prohibición de publicar sondeos electorales en los medios de comunicación cinco días antes de las elecciones o de difundir propaganda durante la denomina jornada de reflexión, en un momento en el que la información se propaga rápidamente a través de internet y las redes sociales, son buena muestra de un marco legal 
que se aprecia desactualizado. Por este motivo, en los últimos años varias voces se levantan a favor de una nueva regulación de la comunicación de las campañas electorales en internet (Campos Domínguez, Valera Ordaz y López García, 2015, p. 1623). Como señala Holgado González (2017), el hecho de que los medios escritos puedan celebrar debates transmitiendo señal por internet, que los ciudadanos se hagan eco a través de las redes sociales de sondeos publicados en medios de comunicación extranjeros, por señalar algunos ejemplos, pone en evidencia la efectividad de una normativa que quizás no se acomoda bien a las nuevas circunstancias y tenga por ello que ser revisada. (p. 461)

Precisamente, uno de los aspectos criticados es la ausencia de los debates electorales televisados en la normativa (Holgado González, 2017), a pesar de su atractivo e importancia en las campañas electorales de cualquier país (Téllez, Muñiz y Ramírez, 2010; Mateos-Crespo, 2017; Navarro Marchante, 2019). La mención a estos espacios solo figura en la legislación de cinco comunidades autonómicas: Andalucía, Murcia, País Vasco, Castilla y León, y Cataluña. Estas carencias se traducen en que la organización de estos encuentros dependa, casi en exclusiva, de la voluntad de los candidatos. Aunque no hay obligación ni prohibición de celebrar debates electorales, cuando se programan es necesario cumplir con unas obligaciones mínimas: 1. Deben comunicarlo previamente a la Junta Electoral Central, con cinco días de antelación (Rúas Araújo, Fernández Holgado y Alén Amil, 2018, p. 162); 2. Los medios de titularidad pública y las televisiones privadas deben respetar los principios de neutralidad, igualdad, proporcionalidad y pluralismo informativo, mientras que las emisoras de radio - que, solo pueden difundir debates, pero no organizarlos -, únicamente están sujetas a los principios de pluralismo e igualdad (Fernández de Casadevante Mayordomo, 2016, p. 212); 3. Finalmente, los medios de comunicación están obligados a compensar a los candidatos de las formaciones políticas con representación excluidas de los debates en contra de su voluntad (Holgado-González, 2017, pp. 475-476).

\subsection{Campaña electoral y redes sociales}

Con el objetivo de comprobar en qué medida las redes sociales han sido incorporadas a los planes de cobertura electoral de las televisiones, se han solicitado estos documentos a los siete entes públicos analizados (La 7 Castilla y León, por ser de titularidad privada, no está obligada a presentar este documento ante la Junta Electoral Provincial). Aunque todos ellos hacen alusión a la posible celebración de un debate electoral, sólo dos mencionan expresamente las redes sociales en el plan, el de Extremadura y el de Castilla-La Mancha. En la propuesta del ente extremeño se indica que "en las redes sociales de Canal Extremadura no se generarán contenidos específicos de campaña para no interferir en el reparto de tiempos asignados a las formaciones políticas". Otro modelo es el de la corporación castellanomanchega, que señala que "los medios digitales de Castilla-La Mancha Media reproducirán en su página web y redes sociales exactamente el mismo contenido informativo emitido por la Televisión Autonómica". Se trata de distintos modos de actuar, aunque en ambos casos los planteamientos son lo suficientemente laxos como para dar libertad a la hora de diseñar patrones de actividad en la retransmisión de contenido electoral.

En lo que se refiere a autorregulación, aunque hay varias televisiones que mencionan las redes sociales en sus libros de estilo -Corporació Catalana de Mitjans Audiovisuals, Corporación Radio e Televisión de Galicia, Corporació Valenciana de Mitjans de Comunicació y Euskal Irrati Telebistasólo la corporación vasca cuenta con un apartado específico sobre redes sociales en tiempo de 
campaña. En él se señala que "el uso de redes sociales debe someterse a los mismos criterios de independencia, imparcialidad y equilibrio informativo. EiTB promoverá la participación ciudadana y el debate con los candidatos y candidatas a través de las redes sociales y fomentará los formatos que favorezcan la interacción con la audiencia". Hay que precisar que la televisión vasca no ha sido considerada en este estudio porque no se celebraron elecciones autonómicas en esta comunidad en mayo de 2019.

En el ámbito internacional destaca el caso de la BBC en Reino Unido, que incorpora manuales específicos de las últimas citas electorales (elecciones locales, estatales y europeas). En ellas se localiza un apartado referido al uso de las redes sociales en el que se indica que el personal de la corporación debe evitar comprometer la imparcialidad de la $\mathrm{BBC}$ expresando sus propios puntos de vista. A pesar de que hay un apartado relacionado con los debates, solo se remarca la necesidad de consulta previa con el Asesor Principal de Política para considerar los temas a abordar y el tipo de audiencia que estará presente en el plató.

Tanto las desigualdades en las normas y códigos de uso de redes sociales como las voces discordantes respecto a la legislación actual hacen relevante observar el comportamiento de las televisiones en campaña electoral para poder comprobar las prácticas que adoptan. Este trabajo pretende así contribuir al debate sobre la idoneidad de una regulación que el contexto comunicativo actual ha superado en muchos aspectos.

\section{Método}

En los últimos años, el crecimiento exponencial y uso masivo de las redes sociales ha despertado un gran interés desde distintas ramas de conocimiento para investigar las relaciones existentes entre los distintos actores que intervienen en un escenario determinado, así como la naturaleza de esas relaciones (Roig-Vila, Mondéjar y Lorenzo Lledó, 2015). Efectivamente, las redes sociales interconectan personas que comparten intereses o relaciones sociales de distinta índole, como puede ser parentesco, uso de una lengua común, colaboraciones personales y profesionales, intercambio de información o productos, o simplemente colaboración entre diferentes colectivos sociales.

A pesar de que la tecnología usada para crear redes sociales es relativamente nueva (Rueda Ortiz, 2015), las redes de interacción social son primarias y han existido desde siempre. En este sentido, y desde el concepto más amplio del término, se puede considerar una red como una colección de entes y sus relaciones. En esa red, los entes que están conectados se denominan nodos o vértices, y las conexiones entre los nodos se denominan enlaces o "links". Por lo tanto, y de forma más concreta, una red social se forma cuando existe una interacción, directa o indirecta, de unas personas con otras, con instituciones u otros colectivos. El análisis de estas redes sociales se lleva a cabo aplicando un conjunto de metodologías matemáticas que se enmarcan en el campo de la teoría de grafos. Así, el análisis de redes nos permite visualizar grandes cantidades de interrelaciones complejas mediante mapas, grafos o sociogramas de símbolos conectados, y poder calcular sobre esos mapas medidas concretas del tamaño, forma y densidad de la red, así como la relevancia de cada nodo.

\subsection{Características de la muestra}

Para monitorizar la conversación generada durante cada uno de los ocho debates televisados se han utilizado las etiquetas promovidas por las televisiones para promocionar el programa especial. Los espacios se emitieron en el seno de la campaña electoral de las elecciones autonómicas del 26 de mayo, entre el 14 de mayo y el 21 de mayo. Se ha excluido IB3 (Baleares) emitido el 21 de mayo, ya que la cadena no utilizó un hashtag, lo que impidió recabar el tráfico específico de la conversación en 
torno al debate. Por otro lado, se han tenido en cuenta en la muestra La 7 (Murcia), que mantiene una gestión indirecta, y la Televisión de Castilla y León, de gestión privada, con la intención de detectar si se registran diferencias respecto a los entes públicos, como un menor control o un uso diferente de las redes sociales.

Mediante el filtrado de la etiqueta, se han capturado todos los tuits generados desde 24 horas antes de la emisión del espacio televisivo hasta 24 horas después, extrayendo en cada caso las interacciones generadas, ya que, de acuerdo a los objetivos, lo relevante es el intercambio y la relación que se establece entre distintos nodos o cuentas de Twitter. Una vez conformado el corpus completo, con un volumen global de 96.512 interacciones o links entre 13.823 nodos o cuentas, se ha procedido a analizar cada caso de manera individual y comparada.

Tabla 1.

\begin{tabular}{|c|c|c|c|}
\hline & Perfill Twitter & Fecha debate & Hashtag \\
\hline ARAGÓN TV & @aragontv & $14 / 05$ & \#DebateATV26M \\
\hline RTVC (CANARIAS) & @,RTVCes & $15 / 05$ & \#RTVCDebate \\
\hline LA 7 (MURCIA) & (a)La7 tv & $17 / 05$ & \#7TVDebate \\
\hline TELEMADRID & @)telemadrid & $19 / 05$ & \#ElDebateMadridVota \\
\hline $\begin{array}{l}\text { CASTILLA-LA MANCHA } \\
\text { MEDIA }\end{array}$ & (a)CMM_es & $20 / 05$ & \#ElDebateCMM \\
\hline TPA (ASTURIAS) & @,RTPAOficial & $21 / 05$ & \#debateTPA \\
\hline CANAL EXTREMADURA & (a)cextremadura & $21 / 05$ & \#ExtremaduraDecide \\
\hline TV CASTILLA Y LEÓN & artvcyl & $21 / 05$ & \#ElDebateCyLTV \\
\hline
\end{tabular}

Fuente: elaboración propia a partir de los datos localizados en los portales corporativos de las televisiones y en sus cuentas de Twitter.

\subsection{Procedimiento para la extracción de tweets y formación de los sociogramas}

Conformada la muestra, la extracción de tuits y análisis posterior se llevó a cabo a través del software comercial Node XL, que proporciona un conjunto de herramientas programadas en lenguaje Visual $\mathrm{C}++$ que permite el análisis de redes sociales a través de la formación de grafos o sociogramas, utilizando el programa Microsoft Excel como base para el análisis de los datos que forman la red. La recopilación de tuits se llevó a cabo utilizando el interfaz de programación de Twitter, de tal manera que los tuits que contenían el hashtag seleccionado fueron importados a NodeXL en forma de dos hojas de cálculo, una correspondiente a nodos y otra a enlaces. La primera aporta información de las cuentas de Twitter que intervienen en el debate; la segunda muestra, en forma de pares de nombres, el origen y destino del mensaje, junto con otros atributos adicionales e información sobre esa relación. En este sentido, es importante destacar que las interrelaciones producidas en Twitter tienen un carácter dirigido, es decir, los tuits, menciones o retuits se muestran en Node XL como un par de actores, correspondientes con el origen y el destino del mensaje, respectivamente. Por último, una vez procesados los datos, se procedió a la formación del modelo matemático que representa las relaciones entre los distintos actores del sociograma, y se procedió al cálculo de las métricas de centralidad sobre el grafo generado.

\subsection{Cálculo de métricas de centralidad}

De acuerdo a los objetivos de este estudio, lo que interesa no son las características de cada nodo o actor en sí mismo, sino de qué manera se relaciona con otros en la red. En este sentido, científicos de datos, matemáticos y sociólogos han colaborado a lo largo de los años para crear metodologías que 
permitan calcular métricas de análisis de redes, creando bases numéricas que permitan comparar y analizar patrones de comportamiento, y estudiar su evolución a lo largo del tiempo. Para algunos autores, la difusión de un mensaje es proporcional a la conectividad de los usuarios, es decir, "cuanto más alto sea el valor de la centralidad calculado para un nodo, más importante es dicho nodo para la conectividad de la red y, por tanto, para la difusión de la información" (Arcila, Barredo y Castro, 2017, p. 69). En este sentido, las medidas de centralidad han ido cobrando mayor relevancia en los últimos años, ya que permiten reflejar cómo de importante (central) es un nodo dentro de la red basado en criterios objetivos y cuantificables. Como han corroborado otros estudios previos, el número de seguidores o perfiles seguidos resulta menos determinante en la difusión de los mensajes que la actividad de los usuarios y su posición en la red de menciones (Toledo Bastos; Galdini Raimundo y Travitzki, 2013).

En este trabajo, para analizar cuáles son los actores más relevantes (nodos dentro de la red) de la conversación, se han calculado diferentes medidas de centralidad: el grado de centralidad, la centralidad de intermediación y la centralidad propia para cada caso en estudio.

\subsubsection{Grado de centralidad (Degree centrality)}

El grado de centralidad es el número de links (menciones) que están conectados a un nodo (perfil de Twitter). Así, se pueden distinguir dos tipos de grados de centralidad: el grado hacia dentro (Indegree centrality) se define como el número de menciones que otros actores en la red hacen sobre una cuenta concreta; el grado hacia afuera (Out-degree centrality) se define como el número de menciones que una cuenta produce. Es, por tanto, una medida de la actividad de un actor dentro de la red.

\subsubsection{Centralidad de intermediación (Betweeness centrality)}

La centralidad de intermediación es una medida que cuantifica lo importante que es un actor dentro del debate a la hora de mantener comunicados a los diferentes nodos (usuarios) de la red conversacional. Se puede afirmar que existen nodos clave en la red, que sirven de puente entre distintos colectivos, de tal forma que, si no existieran, esos grupos de usuarios quedarían aislados de la red. Por lo tanto, la centralidad de intermediación cuantifica lo importante que es un vértice dentro del debate a la hora de mantener comunicados a los diferentes nodos de la red. Desde un punto de vista metodológico, la centralidad de intermediación cuantifica la frecuencia o el número de veces que un nodo actúa como 'puente' cuando dos nodos cualesquiera de la red se comunican entre ellos a través del camino más corto. En otras palabras, cuanto más alto es el valor de centralidad de intermediación, más importante es el nodo como elemento de conexión entre distintos usuarios.

\subsubsection{Centralidad propia (Eigencentrality)}

Esta medida parte de la premisa de que mencionar a un perfil popular es más relevante que hacer una mención a un nodo solitario, aislado y de poca relevancia dentro de la red. Esta métrica tiene en cuenta no sólo las menciones a un perfil sino además la popularidad de los usuarios que lo mencionan. Por lo tanto, un alto coeficiente de centralidad propia significa que ese nodo está a su vez conectado a muchos nodos que también son influyentes. Algunos algoritmos como el Page Rank de Google usan una variante de esta medida para medir la relevancia de las páginas web en internet. 


\section{Resultados}

\subsection{Audiencia tradicional, audiencia en Twitter y evolución temporal de la conversación}

En la comparativa global del tráfico generado por cada uno de los ocho hashtags, se comprueba que los resultados alcanzados en los debates de las distintas comunidades autónomas son muy desiguales. En la tabla 2 se detallan los siguientes parámetros: conjunto de menciones realizadas por la cuenta de cada televisión, número de cuentas que ha mencionado el perfil, las interacciones (links) que se generaron utilizando el hashtag y el número de actores implicados en la conversación.

Tabla 2.

\begin{tabular}{|c|c|c|c|c|c|c|}
\hline Cuenta & Seguidores & Share & $\mathbf{N}^{0}$ menciones & $\begin{array}{l}\mathbf{N}^{0} \text { cuentas } \\
\text { mencionadas }\end{array}$ & $\begin{array}{l}\text { Interacciones } \\
\text { generadas }\end{array}$ & $\begin{array}{l}\text { Actores } \\
\text { implicados }\end{array}$ \\
\hline @aragontv & 69.566 & $6,9 \%$ & 24 & 11 & 11.570 & 876 \\
\hline @RTVCes & 48.874 & $6,1 \%$ & 1 & 1 & 2.225 & 579 \\
\hline @La7_tv & 18.307 & $2,5 \%$ & 8 & 10 & 4.413 & 628 \\
\hline @telemadrid & 80.975 & $7,9 \%$ & 119 & 26 & 34.444 & 7.306 \\
\hline @CMM_es & 39.359 & $9,2 \%$ & 130 & 17 & 21.106 & 1.696 \\
\hline @RTPAOficial & 2.769 & $8,8 \%$ & 3 & 1 & 1.572 & 302 \\
\hline @cextremadura & 68.531 & $9,3 \%$ & 39 & 10 & 5.984 & 908 \\
\hline (a)rtvcyl & 57.993 & $3.3 \%$ & 223 & 16 & 15.198 & 1.528 \\
\hline
\end{tabular}

Fuente: elaboración propia. Datos de audiencia difundidos por Castilla-La Mancha Media y muestra recogidas mediante el interface de programación de Twitter.

Los datos indican que una mayor audiencia televisiva no siempre supone un mayor tráfico de tuits durante los debates electorales: las televisiones que alcanzaron mayor cuota de share (Canal Extremadura, Castilla-la Mancha Media y Televisión del Principado de Asturias) no se corresponden con aquellas que generaron un debate más activo en Twitter, excepto en el caso de Castilla La Mancha Media que aparece bien posicionada en ambos parámetros. Por otro lado, se constata, en coincidencia con otros estudios mencionados, que un mayor número de seguidores en el perfil tampoco se traduce en un mayor tráfico durante los debates. Sólo la televisión pública madrileña se sitúa a la cabeza en número de seguidores, número de interacciones y número de cuentas mencionadas. En cambio, televisiones como la de Canarias, con un número de seguidores muy notable, no actuó como dinamizadora del encuentro, utilizando el hashtag únicamente para promocionar el espacio, recordando el día y la hora de emisión. La infrautilización de este canal se traduce en un número muy bajo de interacciones, lo que indica que no se produjo una dialéctica intensa y participativa durante su emisión.

En relación con ello, un dato significativo es que sí parece existir correlación entre el número de cuentas mencionadas por el perfil oficial de la televisión y la cantidad de tráfico generado por el hashtag. En otras palabras, cuando la televisión es activa haciendo uso del hashtag y mencionando a más actores, logra implicar a más cuentas en la conversación y también potencia su interrelación. Así se confirma en el caso de Telemadrid, Castilla-La Mancha Media y La 7 de Castilla y León, televisiones que, como se verá en los grafos, ocuparon un lugar relevante en la red conversacional de Twitter. 


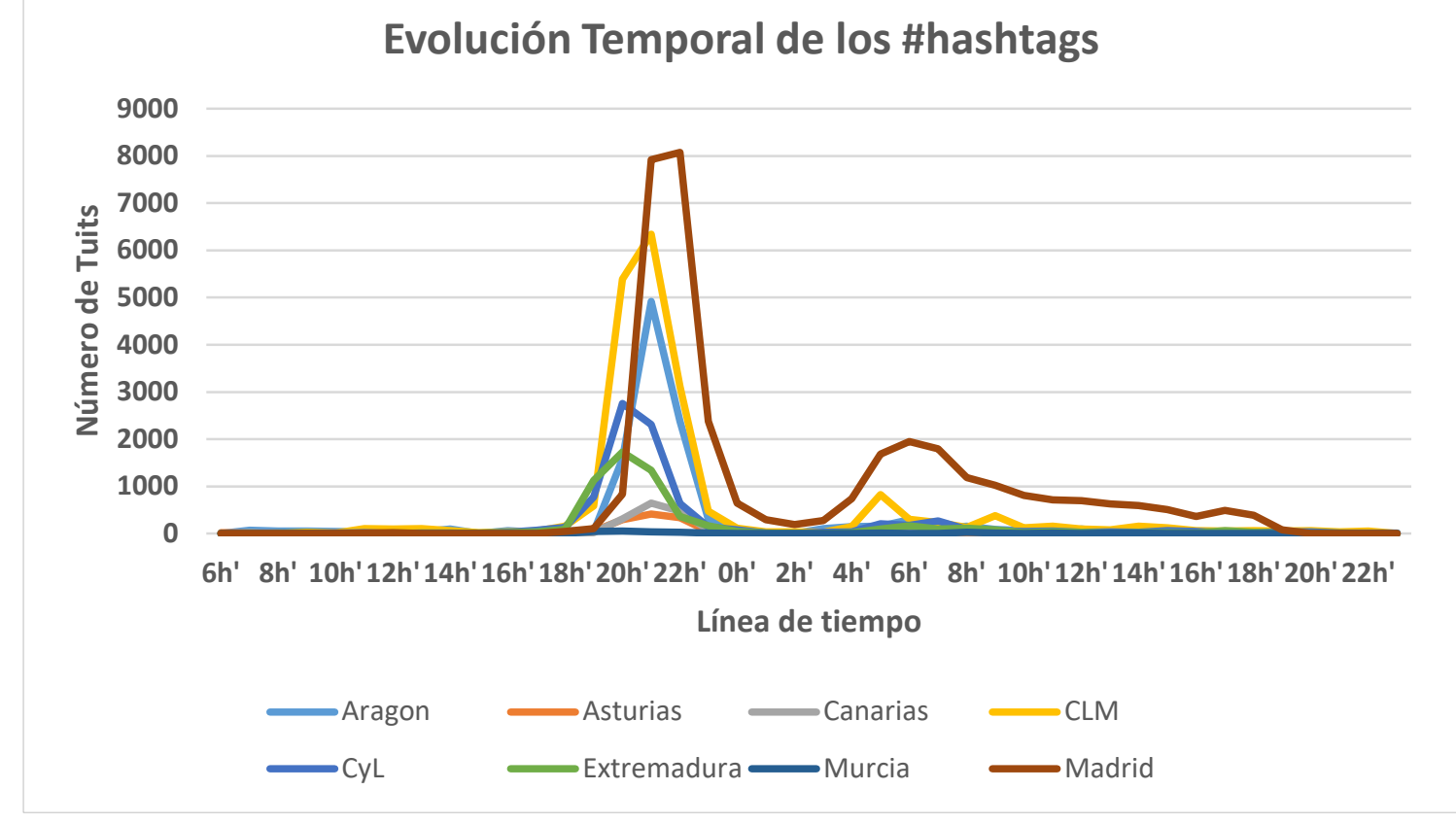

Gráfico 1. En el gráfico comparativo se refleja el tráfico de tuits desde seis horas antes del debate hasta 24 horas después.

Fuente: elaboración propia a partir de la muestra recogida.

Una de las ventajas de explotar el canal de Twitter como medio difusor de este tipo de encuentros es que, terminada la emisión del espacio, la televisión puede continuar la discusión y análisis posterior también en la red social y no sólo desde el plató, como es habitual. En la gráfica comparativa del uso del hashtag entre las ocho televisiones antes y después del debate se observa con claridad que el encuentro celebrado en Telemadrid fue objeto de comentarios varias horas después de su emisión. En concreto, se aprecia que tuvo un pico de interés a primera hora de la mañana, coincidiendo con las tertulias políticas radiofónicas y los magacines televisivos matinales. Así, la televisión madrileña logra que su debate siga teniendo relevancia y generando conversación el día después, algo que también sucede, aunque de manera más tenue, con el hashtag de Castilla-La Mancha Media. En el resto de casos, el tráfico se localiza fundamentalmente de manera paralela a la emisión.

\subsection{Comportamiento del nodo y medidas de centralidad}

\subsubsection{Televisiones centrales: Telemadrid, Castilla-La Mancha Media y La 7 Castilla y León}

Los hashtags de las televisiones públicas de Madrid y de Castilla-La Mancha son los que más interacciones generaron y en ambos casos los grafos muestran que ocuparon un papel central en la conversación, que se muestra más polarizada en el caso del debate castellanomanchego. Así, se aprecia cómo cada nodo o actor interacciona con un grupo de seguidores que se muestran aislados del resto. Algunos de estos actores principales son los candidatos a la presidencia, cuyos perfiles se encuentran entre los diez primeros puestos de las medidas de centralidad, siendo Paco Núñez (PP) y Emiliano García Page (PSOE) los mejor posicionados. Otro aspecto que destaca es que la cuenta de la candidata de Ciudadanos, Carmen Picazo, tiene mucha más relevancia que la de su formación, que desciende a la decimosexta posición en centralidad de intermediación, algo que no sucede en los otros partidos políticos. 
En este caso, destaca la actividad de las cuentas@ccooagentesjccm y @forestales_ccoo a la hora de mencionar cuentas (out-degree), medida en la que ocupan el primer y segundo puesto. Ambos perfiles se ocuparon de las propuestas sobre medio ambiente que recogían los programas electorales de todos los partidos e hicieron activismo a través de una conversación conjunta bajo la etiqueta \#AgentesMedioambientales mencionando una media de 50 usuarios por tuit. La televisión de Castilla-La Mancha dinamizó el debate a través de su cuenta corporativa y también del perfil @ cmm_noticias, cuenta que resultó todavía más relevante a la hora de articular la conversación de las distintas comunidades, ocupando la segunda posición en centralidad de intermediación y la primera en centralidad propia.

En el debate de Telemadrid, las medidas muestran la relevancia de la cuenta de Isa Serra, candidata de la formación Unidas Podemos, que ocupa el primer lugar en centralidad de intermediación y en centralidad propia, siendo el segundo puesto en ambas medidas para la cuenta del candidato de Ciudadanos, Ignacio Aguado, y el tercero para la cuenta de Telemadrid, lo que indica que jugó un papel importante de conectividad entre usuarios y de dinamización de la conversación. Además, es la televisión que logró implicar a más cuentas en la conversación sobre el debate, con 7.303 usuarios que intervinieron de alguna manera en el debate paralelo. En este caso, la densidad con que se muestra la conversación en el grafo revela el elevado número de links generados, síntoma de que suscitó un interés muy alto entre la audiencia social.

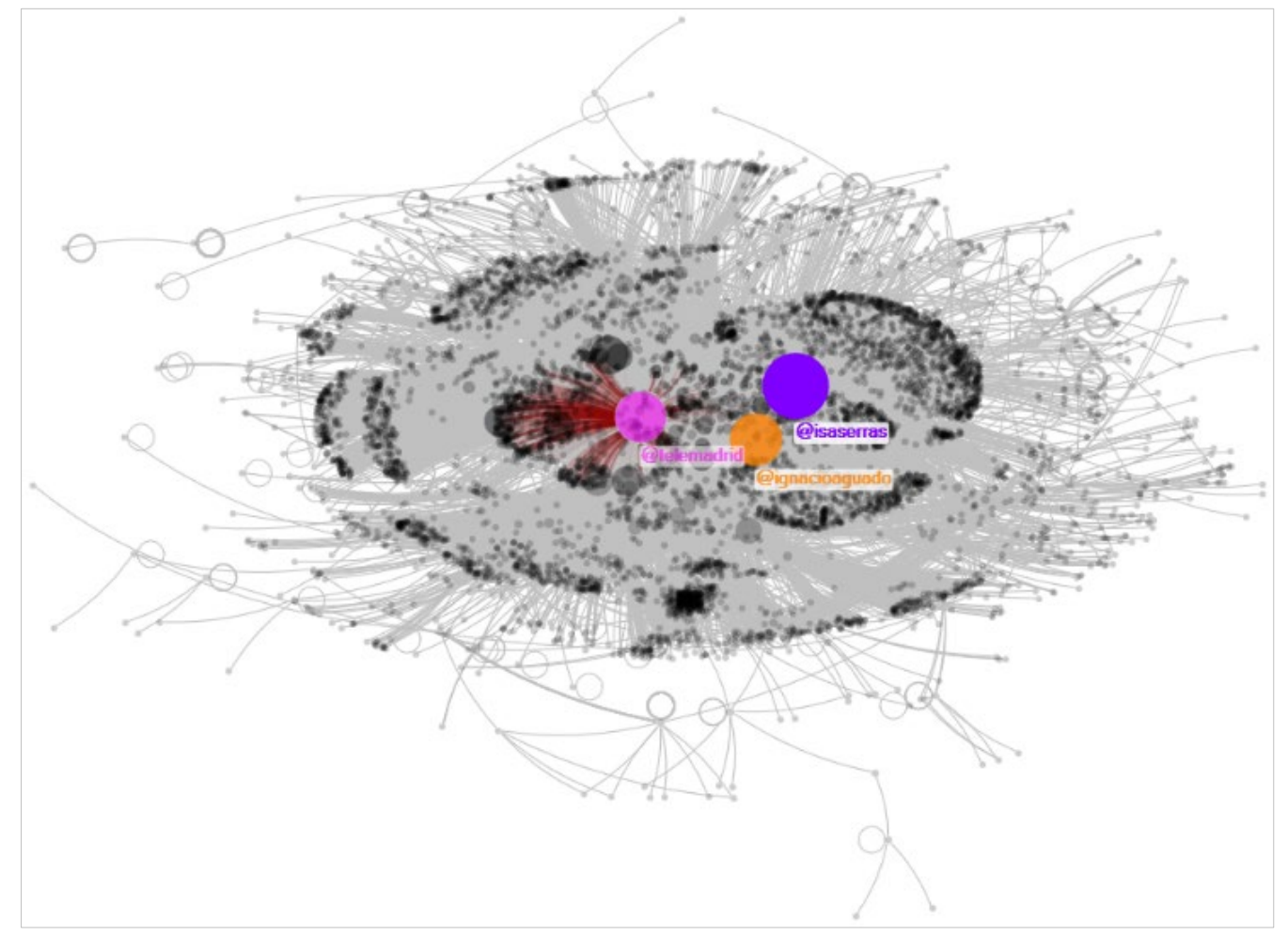

Ilustración 1. Grafo correspondiente al hashtag \#ElDebateMadridVota. Fuente: elaboración propia a partir de la muestra recogida. 


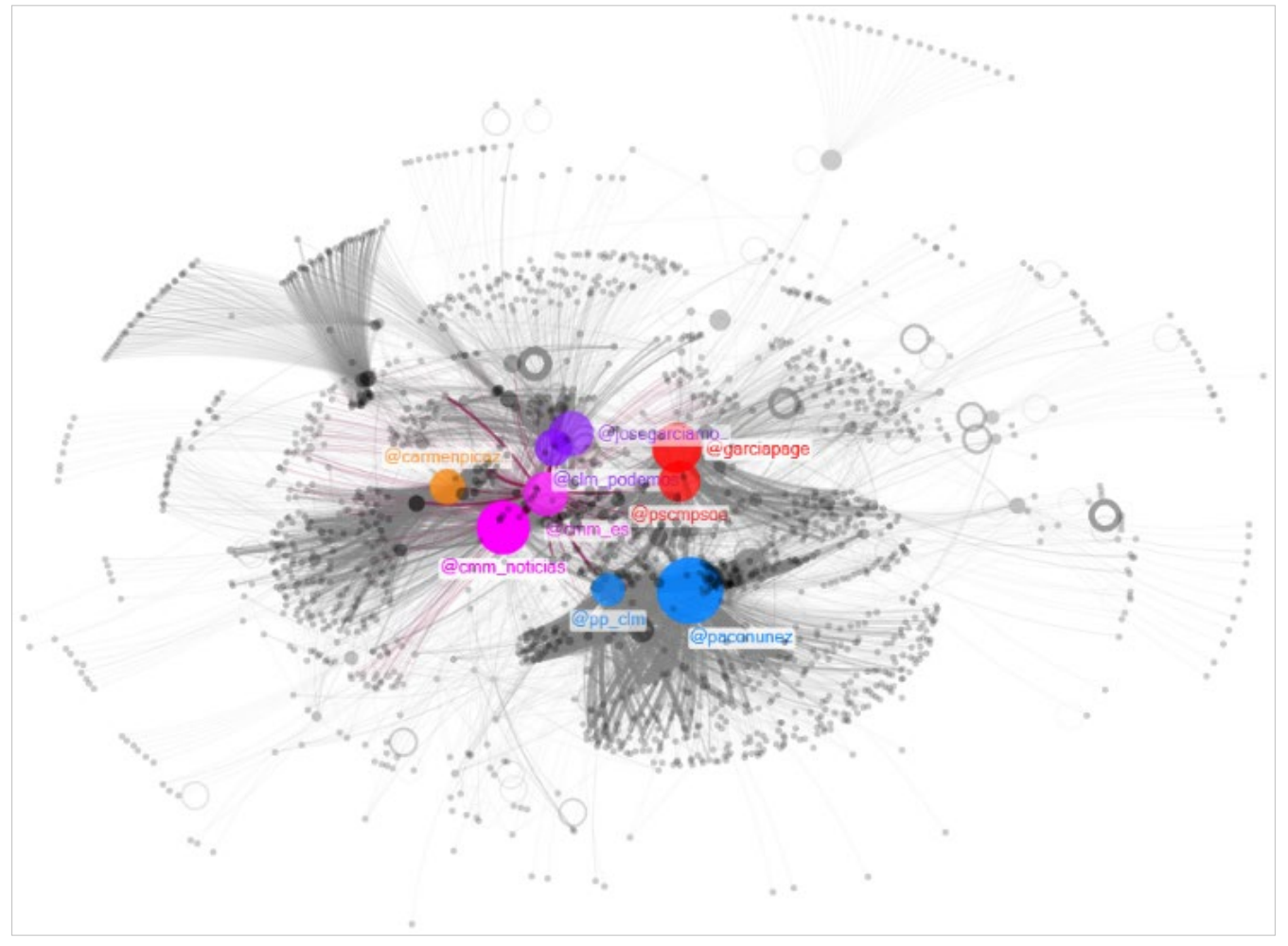

Ilustración 2. Grafo correspondiente al hashtag \#ElDebateCMM.

Fuente: elaboración propia a partir de la muestra recogida.

Un tercer caso significativo es el de la televisión de Castilla y León, que logró 15.198 interacciones. En el grafo se localizan varios nodos principales: las cuentas corporativas de PSOE Castilla y León, y Partido Popular Castilla y León, y sus respectivos candidatos, Luis Tudanca (PSOE) y Alfonso F. Mañueco (PP), y las Pablo Fernández (Podemos Castilla y León) y Francisco Igea (Ciudadanos Castilla y León). En estos dos ejemplos, las cuentas de sus respectivos partidos no alcanzaron mucha notoriedad y la conversación giró en torno a los líderes, lo que indica que PP y PSOE capitalizaron mejor la atención el debate en la red al mover la etiqueta con las cuentas de candidato y partido.

El grafo muestra además el papel central del ente para concentrar la conversación. El perfil corporativo ocupa el cuarto lugar en centralidad de intermediación y el quinto en la centralidad propia, por lo que tuvo una posición significativa como puente entre diferentes colectivos que sin él se encontrarían aislados fraccionando la red en diferentes subredes. Es el candidato de Podemos el que se muestra más alejado del núcleo central de la conversación y el que quedaría al margen sin el papel de moderador/puente que ejerce la cuenta de la televisión castellano y leonesa. 


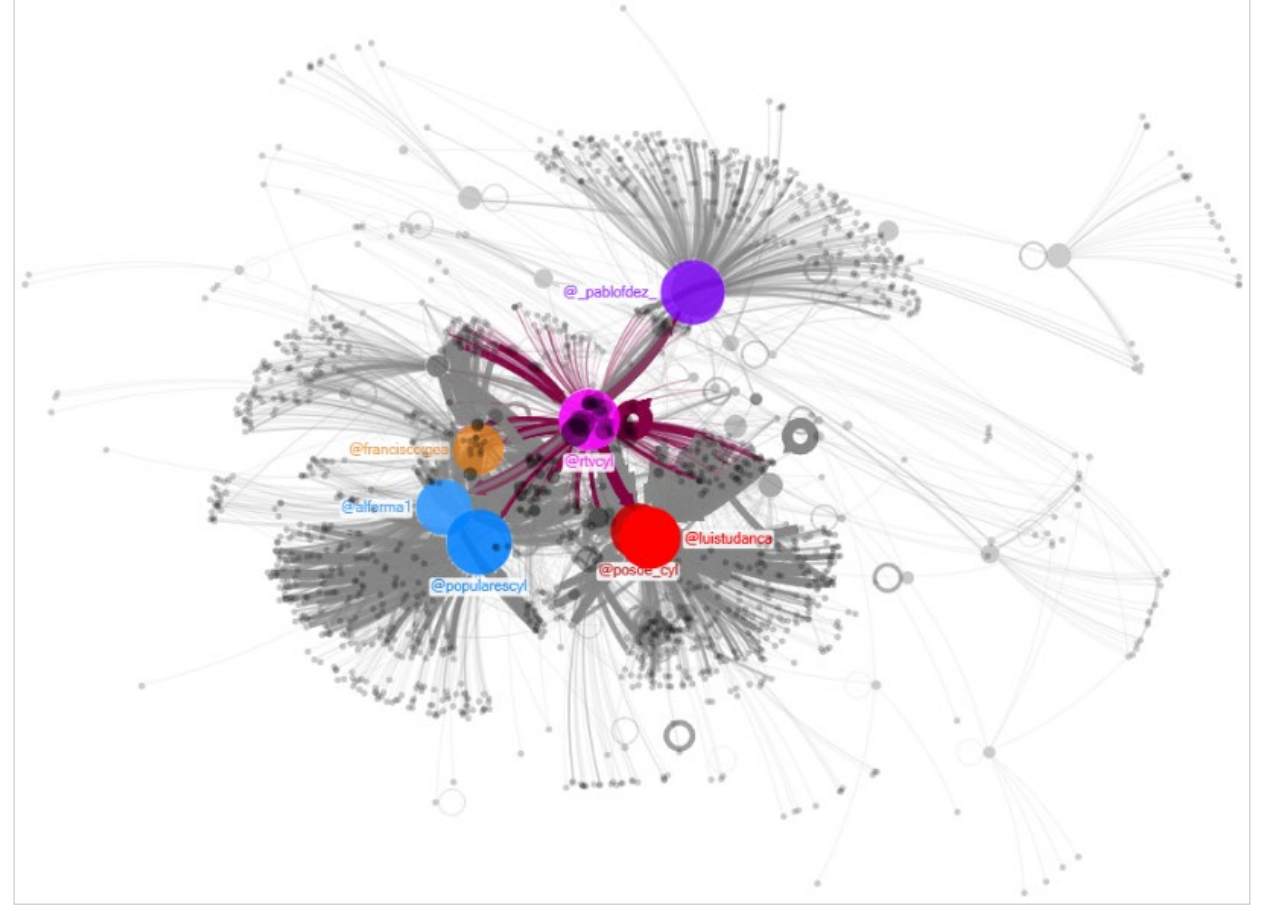

Ilustración 3. Grafo correspondiente al hashtag \#ElDebateCyLTV.

Fuente: elaboración propia a partir de la muestra recogida.

Los tres casos son una muestra de buen uso de Twitter para canalizar el debate en la segunda pantalla y confirman la centralidad alcanzada por la cuenta de la televisión regional (pública en Madrid y Castilla-La Mancha; privada en Castilla y León) durante la emisión de su debate.

En cuanto a la actividad de las cuentas de las televisiones, se puede decir que mantuvieron un nivel alto de interacciones: 119 interacciones y 26 cuentas mencionadas en el caso de Telemadrid; 130 y 17 cuentas mencionadas en el caso de Castilla-La Mancha Media; y 223 y 16 cuentas mencionadas por parte de La 7 Castilla y León. En la Tabla 3 se puede ver la distribución de menciones a partidos políticos y candidatos que hicieron las televisiones en Twitter.

Tabla 3. Menciones realizadas por la cuenta de la televisión.

\begin{tabular}{|l|l|l|l|l|l|}
\hline \multicolumn{2}{|l|}{$\begin{array}{l}\text { Castilla-La Mancha } \\
\text { Telemadrid }\end{array}$} & \multicolumn{2}{l|}{ La 7 Castilla y León } \\
\hline equipogabilondo & 11 & pp_clm & 10 & pablofdez__ & 33 \\
\hline idiazayuso & 10 & pscmpsoe & 10 & alferma1 & 33 \\
\hline ignacioaguado & 10 & vox_es & 10 & franciscoigea & 33 \\
\hline ppmadrid & 9 & cs_clm & 10 & luistudanca & 31 \\
\hline cs_madrid & 9 & clm_podemos & 9 & ciudadanoscs & 12 \\
\hline monasterior & 9 & paconunez & 6 & psoe_cyl & 10 \\
\hline isaserras & 8 & carmenpicazocs & 6 & podemos_cyl & 10 \\
\hline madrid_vox & 7 & garciapage & 6 & popularescyl & 10 \\
\hline psoe_m & 7 & dariveg & 6 & & \\
\hline podemoscmadrid & 5 & josergarmolina & 5 & & \\
\hline
\end{tabular}

Fuente: elaboración propia a partir de los datos recogidos en la muestra.

Los datos confirman una menor atención a Podemos por parte de Telemadrid (13 menciones entre candidata y formación, frente a las 19 que obtuvo el Partido Popular), y unas dinámicas de 
menciones bastante equitativas en el caso de las otras dos televisiones, donde apenas se producen diferencias entre candidatos y partidos políticos. Entre los mencionados por la televisión castellano y leonesa no está la formación Vox, si bien el candidato de la formación política no participó en el debate.

Por otro lado, Telemadrid no sólo mencionó a las diez cuentas reseñadas en la tabla; también mencionó a catorce cuentas que no pertenecían a partidos políticos, entre las que se encontraban nueve periodistas o comentaristas de la cadena que participaron en la retransmisión. Estos datos ponen de relieve que se hizo una apuesta por estimular el diálogo en las redes sociales e incluso hacer verificación de datos en directo, ya que otras cuentas mencionadas fueron Maldito Dato y Maldita hemeroteca, y la de su fundadora, Clara Jiménez Cruz. A pesar de ello, frente a las 26 menciones realizadas, la cuenta de Telemadrid fue mencionada por 927 personas, lo que indicaría que no se produjo un intercambio comunicativo entre el ente y los espectadores, sino que Twitter se utilizó básicamente como un canal emisor de contenido.

\subsubsection{El caso intermedio: Aragón TV y Canal Extremadura}

Aragón TV y Canal Extremadura muestran una actividad menor y estrategias similares, con 11 cuentas mencionadas en el primer caso y 10 en el segundo. La televisión aragonesa baja al décimo puesto en centralidad de intermediación y al sexto en centralidad propia, por lo que resulta menos relevante en cuanto a conectividad de los usuarios e importancia en el debate. En este caso, el núcleo central de la conversación es claramente Javier Lambán (PSOE). Otros actores importantes fueron Luis María Beamonte (PP) y Arturo Aliaga (PAR), mientras que en Podemos y Ciudadanos las cuentas relevantes fueron las de los partidos.

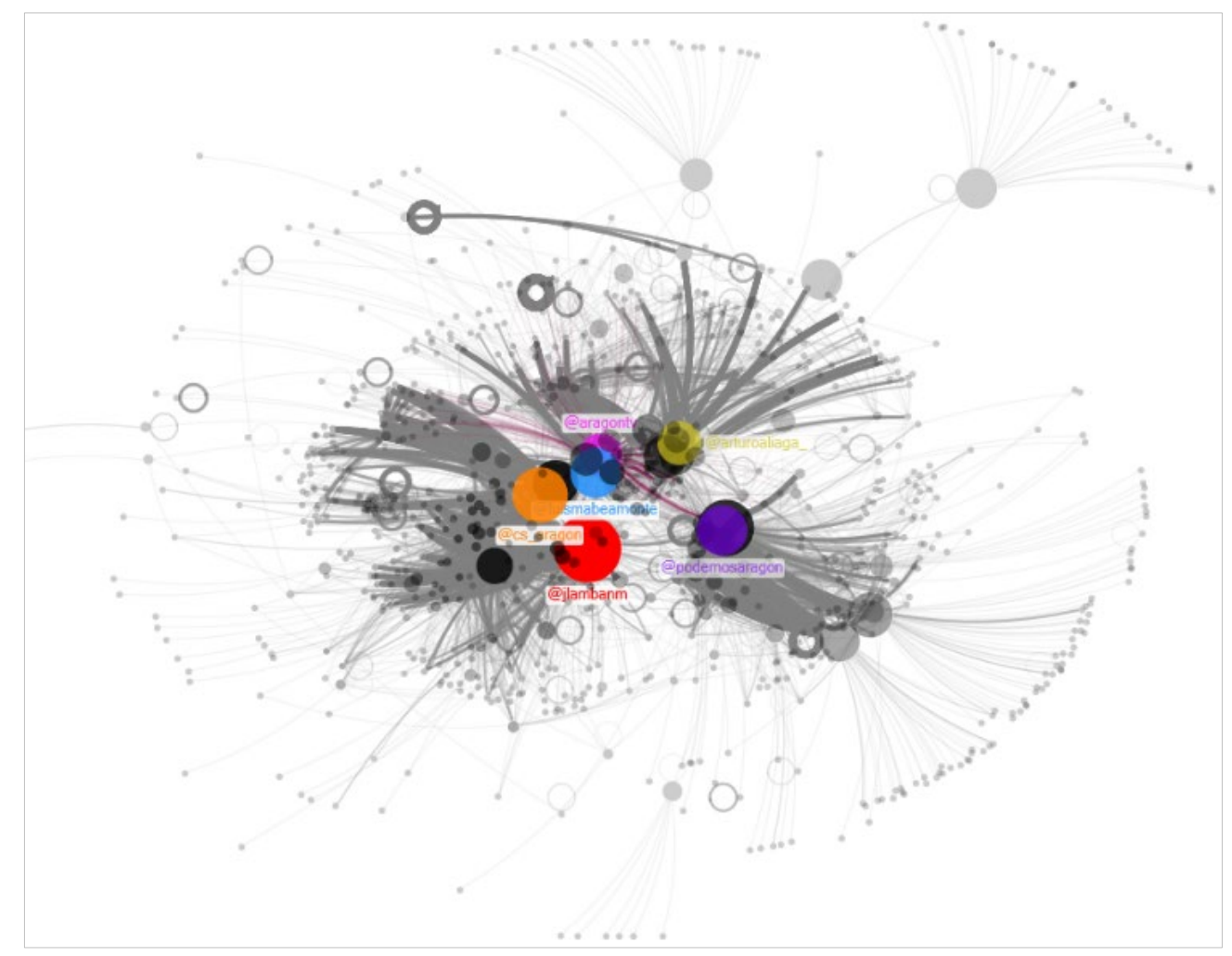

Ilustración 4. Grafo correspondiente al hashtag \#DebateATV26M.

Fuente: elaboración propia a partir de la muestra recogida. 


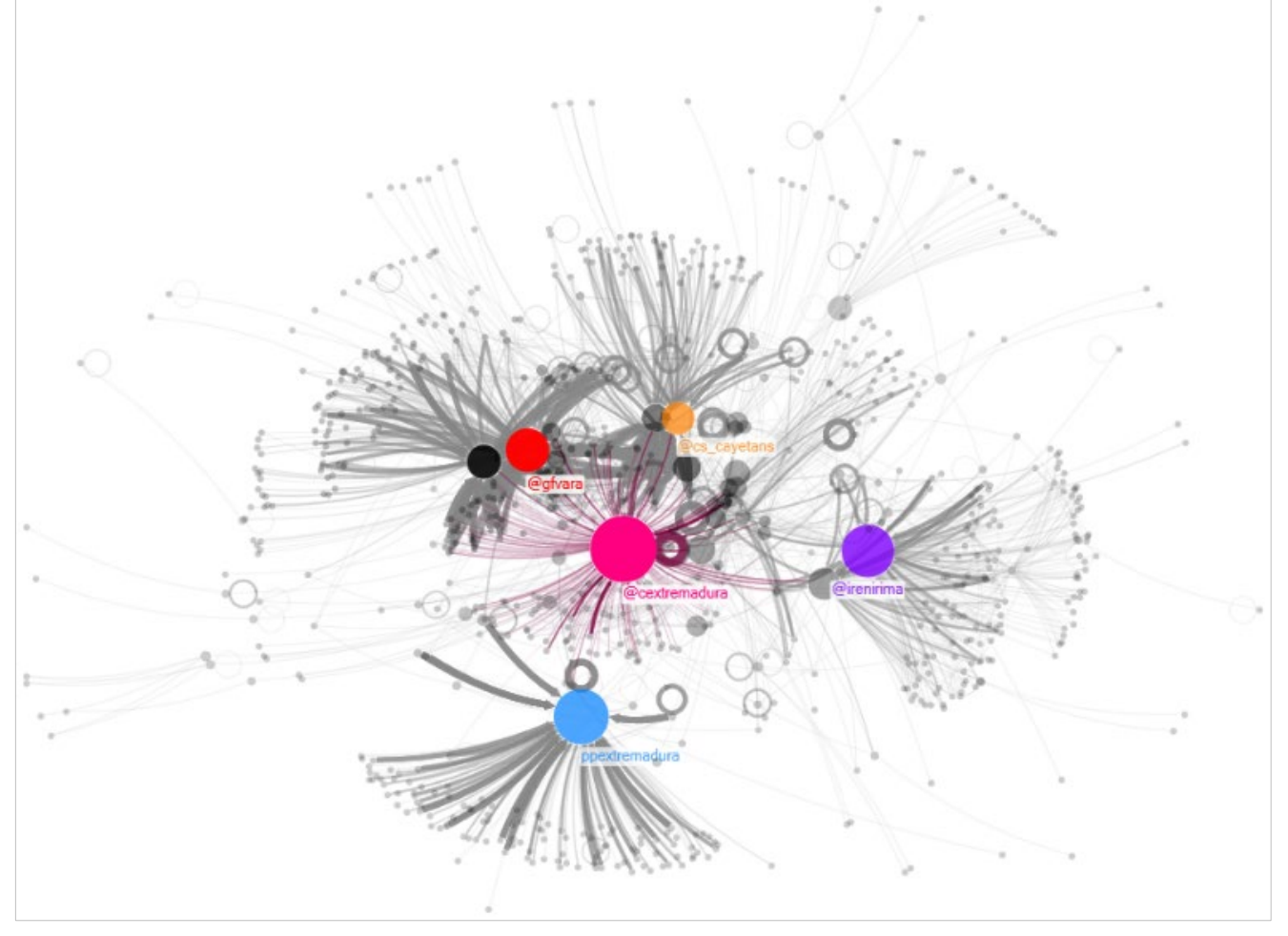

Ilustración 5. Grafo correspondiente al hashtag \#ExtremaduraDecide. Fuente: elaboración propia a partir de la muestra recogida.

En el caso de Extremadura, la televisión se encuentra en primer lugar en ambos parámetros resultando el núcleo central de la conversación, aunque su hashtag sólo produce 5.984 links, cifra muy alejada de los tres casos anteriores que superaban las 15.000 interacciones. En este sentido, un aspecto a considerar es que no es lo mismo registrar un alto grado de centralidad en una conversación de apenas un millar de interacciones que en una que logró la participación muchos usuarios muy activos.

Al revisar las dinámicas de difusión en Twitter de la cuenta oficial @cextremadura durante el debate, se comprueba que el $74 \%$ de los tuits no incorporaron ninguna mención. Además, no todos los candidatos (7) que participaron en el debate televisado fueron mencionados. La cuenta nombró una vez a Guillermo Fernández Vara, del PSOE; Juan Antonio Morales, de Vox; y Cayetano Polo, de Ciudadanos, mencionando también las cuentas de sus respectivas formaciones políticas. En cambio, no figuran entre las menciones tres candidatos que no tienen perfil de Twitter - José Antonio Monago (PP), Pedro Lanzas (Extremadura Unida) y Laura Márquez (Actúa) - ni tampoco Irene de Miguel (Podemos) ni su formación, a pesar de que su cuenta, @irenirima, fue un nodo central en el debate paralelo, como confirma su segundo puesto en el número de menciones recibidas (in-degree), el tercero en centralidad de intermediación y el séptimo en centralidad propia. De hecho, el grafo refleja que tanto Podemos como el Partido Popular se sitúan de alguna manera al margen del núcleo de la conversación (Canal Extremadura), estableciendo conexiones únicamente con sus seguidores.

Aragón televisión, por su parte, menciona únicamente a cuentas de candidatos, no a partidos políticos, y lo hace de manera equitativa, con dos menciones a cada uno de los ocho líderes que participaron en el encuentro televisivo. 
RLCS, Revista Latina de Comunicación Social, 76, 97-119

[Investigación] DOI: 10.4185/RLCS-2020-1439 | ISSN 1138-5820 | Año 2020

Tabla 4. Menciones realizadas por la cuenta de la televisión.

\begin{tabular}{|l|l|l|l|}
\hline \multicolumn{2}{|l|}{ Aragón TV } & \multicolumn{2}{l|}{ Canal Extremadura } \\
\hline s1moron & 2 & Psoeex & 1 \\
\hline asanzr & 2 & juan_a_morales & 1 \\
\hline arturoaliaga_ & 2 & ppextremadura & 1 \\
\hline danielperezcs & 2 & csextremadura & 1 \\
\hline marudaz1 & 2 & cs_cayetans & 1 \\
\hline pepesoro & 2 & Gfvara & 1 \\
\hline luismabeamonte & 2 & & \\
\hline jlambanm & 2 & & \\
\hline
\end{tabular}

Fuente: elaboración propia a partir de los datos recogidos en la muestra.

\subsubsection{Televisiones sin actividad relevante: Televisión Canaria, La 7 (Murcia) y TPA (Asturias)}

En estos tres casos, la actividad de la televisión en Twitter fue muy escasa, lo que derivó en un número de interacciones mucho más bajo que el obtenido con los demás hashtags. La Televisión del Principado de Asturias y Televisión Canaria adoptaron la estrategia de mencionarse solo a sí mismas. En el caso de la televisión murciana, La 7 , sí se produce un mayor número de menciones -10 cuentas- pero la cuenta corporativa no hizo uso de hashtag durante el debate y solo lo utilizó a modo de reclamo previo. El medio abrió un hilo explicando las normas, bloques y dinámicas que se iban a seguir y difundió un recordatorio antes de empezar. A pesar de no emitir tuits, logró un volumen de tráfico superior a los otros dos casos, con 4.413 interacciones.

Aunque los grafos no resultan significativos, se muestra como ejemplo el caso de la etiqueta \#debateTPA, del debate asturiano, donde se puede ver una conversación dispersa y poco conectada, en la que apenas destaca la actividad de Adrián Barbón, candidato a la presidencia por el Partido Socialista, Podemos Asturies y la cuenta de la Federación Socialista Asturiana. Aunque la cuenta del ente asturiano se situó en el centro del debate, como demuestran las medidas en el ranking de centralidad de intermediación ( $2^{\circ}$ puesto) y centralidad propia ( $3^{\circ}$ puesto), el tráfico total que logró generar la etiqueta fue muy pequeño, con 1.572 interacciones. Esto viene a corroborar que ser central no lo es todo para lograr relevancia, si no va a acompañado de muchos actores hablando sobre el contenido emitido. 


\section{Ilustración 6. Grafo correspondiente al hashtag \#debateTPA.}

Fuente: elaboración propia a partir de la muestra recogida.

Por otro lado, con el hashtag \#RTVCDebate se generaron 2.225 interacciones pero el perfil de la televisión canaria en este caso ocupa posiciones inferiores en el ranking de centralidad $\left(9^{\circ}\right.$ lugar en centralidad de intermediación y $7^{\circ}$ en centralidad propia), por lo que fue un actor irrelevante. Como ya se ha señalado, la televisión emitió un único tuit anunciando el debate. El núcleo central de la conversación durante esta emisión estuvo en la cuenta@podemoscanarias, en el primer puesto del ranking en los dos parámetros mencionados y también en número de veces mencionado (in-degree).

\section{Discusión y conclusiones}

En 2019 los entes de radiotelevisión de proximidad han apostado por los debates, una iniciativa de servicio público que requiere un esfuerzo de producción, tanto en recursos técnicos como humanos. Sin embargo, sus emisiones han cosechado resultados dispares. Los datos de share muestran que la audiencia tradicional varía en las distintas regiones; al mismo tiempo, se constata que no todas las televisiones han explotado igual estos espacios en Twitter. La televisión madrileña, por ejemplo, logró veinte veces más interacciones que la asturiana; la de Castilla-La Mancha, catorce veces más. A la luz de los datos se puede afirmar que se produce una relación entre el uso del hashtag que hace la televisión y el intercambio comunicativo alcanzado en la red social, ya que aquellas televisiones que más cuentas mencionaron consiguieron implicar a más usuarios en el debate y producir más interacciones en conjunto para la etiqueta propuesta.

A pesar de las posibilidades de interacción que presenta, a priori, la audiencia social, apenas es tenida en consideración por la mayoría de las televisiones autonómicas, utilizándose el perfil corporativo en Twitter, en la mayoría de los casos, como una plataforma de autopromoción del programa especial o "para difundir contenido original y no como un canal de comunicación en sí mismo" (RodríguezVázquez et al., 2016). Los resultados ponen de relieve que no existe en la actualidad ninguna pauta sobre las dinámicas de difusión de estos contenidos en la segunda pantalla y cada ente opta por desarrollar su estrategia, que va desde no hacer uso del hashtag propuesto durante la emisión del debate 
(La 7), hasta adoptar un papel muy activo en la retransmisión del espacio, erigiéndose en nodo central de la conversación (Castilla-La Mancha Media, Televisión de Castilla y León y Telemadrid). Un dato a señalar es que la televisión castellano y leonesa, de titularidad privada, no sólo se encuentra entre las que más ha dinamizado el debate en la red, sino que ha resultado equitativa en el número de menciones a los candidatos, si bien este debate se emitió en diferido, lo que ha podido influir en las rutinas de uso de Twitter. La menos ecuánime resulta ser Canal Extremadura.

Por otro lado, también se aprecia que no todos los candidatos políticos ocupan el mismo espacio en el debate digital. Algunos de ellos ni siquiera tienen perfil en Twitter (José Antonio Monago) lo que se traduce en una ventaja para otros candidatos, que son capaces de concentrar el protagonismo en redes sociales durante la emisión. En este sentido, destaca el perfil de Isa Serra (Podemos) e Ignacio Aguado (Ciudadanos), que consiguieron concentrar gran protagonismo alrededor del debate de Telemadrid. Ambos ocupan los primeros puestos en centralidad de intermediación y centralidad propia, lo que indica que fueron puntos clave para conectar usuarios y que, a su vez, los perfiles con los que interaccionaron eran relevantes. En este sentido, tan importante es imponerse en el ejercicio dialéctico televisado como en su réplica digital. Algunos estudios han confirmado que los candidatos que ganan influencia en las redes sociales son aquellos capaces de crear una sinergia entre los medios tradicionales y estos nuevos canales (Karlsen y Enjolras, 2016). Además, las redes sociales favorecen una campaña más personal y menos enmarcada en las siglas del partido, una cuestión que resulta más determinante a la hora de elegir a representantes de organismos territoriales.

Un aspecto que llama la atención es que en el ranking de perfiles que más menciones realizaron a otras cuentas (out-degree) los primeros lugares suelen ocuparlos, en la mayor parte de los casos, usuarios que no son personajes públicos, es decir, corresponden a perfiles de personas particulares que, sin embargo, son muy activas en Twitter y alcanzan notoriedad en la conversación. Es el caso, por ejemplo, de Brujastur, que con apenas 85 seguidores ocupa el primer puesto en cuentas mencionadas con \#ElDebateMadridVota; o la de Anais Gallina, a la cabeza en el mismo parámetro para \#ElDebateCyLTV. De hecho, aunque no es el caso, los medios de comunicación ya se han hecho eco de cuentas falsas creadas por partidos políticos o incluso agencias de marketing para acaparar protagonismo en redes en estos eventos ${ }^{3}$.

El escenario actual sugiere que sería oportuno consensuar ciertas dinámicas de uso de redes sociales para este tipo de espacios televisivos que no forman parte de la programación convencional y están sujetos a una regulación específica. Se observa que los planes de cobertura electoral apenas mencionan las redes sociales, obviando la existencia de estos nuevos canales que, sin embargo, resultan esenciales para amplificar el número de espectadores potenciales del programa y alcanzar al segmento con capacidad de voto que se encuentra más alejado de la televisión convencional, el de los jóvenes (Lago-Vázquez et al., 2016). Las precauciones por mantenerse en el territorio de la imparcialidad pueden estar disuadiendo a los entes a la hora de intentar liderar el debate en Twitter, perdiendo así la oportunidad de convertirse en actores relevantes en todos los soportes. Otra causa puede ser que las audiencias aún siguen teniéndose en cuenta únicamente desde la medición tradicional $\mathrm{y}$, por tanto, los equipos directivos concentran sus esfuerzos en ganar puntos de share, sin sacar partido a sus cuentas de Twitter como reclamo hacia el contenido.

Los resultados evidencian que se hace necesaria una renovación de la regulación existente, de forma flexible y combinada con autorregulación y corregulación, para poder adaptarse al cambio que supone la digitalización y las tecnologías (Muñoz-Saldaña y Gómez-Iglesias, 2013), más aún en la cobertura de acontecimientos de especial interés como debates o entrevistas por parte de los medios

${ }^{3}$ Las trampas de los partidos políticos en Twitter, Cadena Ser, Irene Dorta, 21/04/2019. 
de comunicación, en especial los de titularidad pública, bajo una fuerte de sospecha de politización en España (Soengas-Pérez y Rodríguez-Vázquez, 2014; Humanes, Fernández-Alonso, 2015; MarzalFelici y Zallo-Elguezabal, 2016; Soengas-Pérez, Elías-Pérez, López-Cepeda, 2018). Un ejemplo de buena planificación es el de Telemadrid, que demuestra que es posible adoptar una estrategia activa de difusión y emisión de estos espacios en redes sociales. Las vías para hacerlo sin apartarse del marco de neutralidad son, bien a través de una sistemática retransmisión incorporada al plan de cobertura electoral, bien mediante el desarrollo específico de esta cuestión en los libros de estilo. Ambas herramientas de autocontrol son susceptibles de cambio y de renovación, al tiempo que van planteándose nuevos retos tecnológicos.

\section{Referencias bibliográficas}

Arcila, C., Barredo, I. y Castro, C. (2017). Analitica y visualización de datos en Twitter. Editorial UOC.

Asur, S. y Huberman, B. A. (2010). Predicting the future with social media. IEEE/WIC/ACM International Conference on Web Intelligence and Intelligent Agent Technology. Estados Unidos. http://doi.org/10.1109/WI-IAT.2010.63

Campos-Domínguez, E. (2017). Twitter y la comunicación política. El profesional de la información, 26(5), 785-793. http://doi.org/10.3145/epi.2017.sep.01

Campos-Domínguez, E., Valera-Ordaz, L. y López-García, G. (2015). Emisores políticos, mediáticos y ciudadanos en internet: hacia un nuevo marco comunicativo en la jornada de reflexión en España. História, Ciências, Saúde-Manguinhos, (22), 1621-1636. http://doi.org/10.1590/S0104$\underline{59702015000500005}$

Congosto, M. L. y Aragón, P. (2012). Twitter, del sondeo a la sonda: nuevos canales de opinión, nuevos métodos de análisis. Redes Sociales y Gobernanza. Más Poder Local, (12), 50-56.

Congosto, M. L., Deltell-Escolar, L., Claes, F. y Osteso-López, J. M. (2013). Análisis de la audiencia social por medio de Twitter. Caso de estudio: los premios Goya 2013. Revista ICONO14, Revista Científica de Comunicación y Tecnologías Emergentes, 11(2), 53-82.

http://doi.org/10.7195/ri14.v11i2.577

Deltell-Escolar, L., Claes, F. y Osteso-López, J. M. (2013). Audiencias televisivas y líderes de opinión en Twitter. Caso de estudio: El Barco. Estudios sobre el Mensaje Periodístico, 19(1), 347-364. http://doi.org/10.5209/rev ESMP.2013.v19.n1.42526

Fernández-de-Casadevante-Mayordomo, P. (2016). El régimen jurídico de los debates electorales en España. Revista Cuadernos Manuel Giménez Abad, (11), 204-2014.

Giglietto, F. y Selva, D. (2014). Second Screen and Participation: A Content Analysis on a Full Season Dataset of Tweets. Journal of Communication, 64(2), 260-277. http://doi.org/10.1111/jcom.12085

Gómez-Aguilar, M., Paniagua-Rojano, F. J. y Farias-Batlle, P. (2015). Comportamiento de la audiencia de televisión en las redes sociales. Una aproximación al perfil y programas más comentados. Revista Latina de http://doi.org/10.4185/RLCS-2015-1058

Comunicación Social, (70), 539-551. 
Han, E. y Lee, S. W. (2014). Motivations for the complementary use of text-based media during linear TV viewing: An exploratory study. Computers in Human Behavior, (32), 235-243. http://doi.org/10.1016/j.chb.2013.12.015

Harboe, G. (2009). In search of social television. En P. Cesar, D. Geerts y K. Chorianopoulos (Eds.), Social interactive television (pp. 1-13). Idea Group Reference.

Holgado-González, M. (2017). Publicidad e información sobre elecciones en los medos de comunicación durante la campaña electoral. Teoría y realidad constitucional, (40), 457-485. http://doi.org/10.5944/trc.40.2017.20914

Humanes, M. L. y Fernández-Alonso, I. (2015). Pluralismo informativo y medios públicos. La involución de TVE en el contexto del cambio político (2012-2013). Revista Latina de Comunicación Social, (70), 270-287. http://doi.org/10.4185/RLCS-2015-1046

Iñesta-Fernández, N. (2015). Espectador social y convergencia televisiva. Ámbitos. Revista internacional de comunicación, (28), 1-19.

Karlsen, R. \& Enjolras, B. (2016). Styles of social media campaigning and influence in a hybrid political communication system: Linking candidate survey data with Twitter data. International journal of press/politics, 21(3), 338-357. http://doi.org/10.1177/1940161216645335

Lago-Vázquez, D., Direito-Rebollal, S., Rodríguez-Vázquez, A. I. y López-García, X. (2016). El consumo milleniall de información política en televisión y redes sociales. Análisis de la campaña Elecciones Generales en España 2015. Revista Latina de Comunicación Social, (71), 1.151-1.169. http://doi.org/10.4185/RLCS-2016-1139

Larsson, A. O. \& Moe, H. (2011). Studying political microblogging: Twitter users in the 2010 Swedish election campaign. New Media and Society, 14(5), 729-747. http://doi.org/10.1177/1461444811422894

Lochrie, M. \& Coulton, P. (2012). The role of smartphones in mass participation TV. Proceedings of the 10th European conference on interactive TV and Video Conference. ACM, Berlin, Germany, ACM, 199-202.

Marzal-Felici, J. y Zallo-Elguezabal, R. (2016). Presentación: Las televisiones públicas de proximidad ante los retos de la sociedad digital. Comunicación y Sociedad, 29(4), 1-7. http://doi.org/10.15581/003.29.4.sp.1-7

Mateos Crespo, J. L. (2017). La reciente reforma de la LOREG: Una excepcional oportunidad perdida para el cambio de modelo de campaña electoral en España. Anuario de la Facultad de Derecho, (10), 75-102.

Muñoz-Saldaña, M. y Gómez-Iglesias, V. (2013). El protagonismo de la autorregulación y de la corregulación en el nuevo mercado audiovisual digital. Trípodos, (32), 77-92.

Navarro-Marchante, V. J. (2019). Los debates electorales en la televisión: una necesaria revisión de su regulación. Revista española de derecho constitucional, (116). http://doi.org/10.18042/cepc/redc.116.0 
Padilla-Castillo, G. (2015). La espectacularización del debate electoral: estudio de caso en Estados Unidos. Revista de Comunicación Vivat Academia, (132), 162-181.

Quintas-Frouce, N. y González-Neira, A. (2014). Audiencias activas: Participación de la audiencia social en la televisión. Revista Comunicar, (43), 83-90. http://doi.org/10.3916/C43-2014-08

Rodríguez-Vázquez, A. I., Direito-Rebollal, S. y Lago-Vázquez, D. (2016). Estrategias de transcodificación de los géneros televisivos del prime-time español. El profesional de la información, 25(3), 413-422. http://doi.org/10.3145/epi.2016.may.11

Roig-Vila, R., Mondéjar, L. y Lorenzo-Lledó, G. (2015). Redes sociales científicas. La Web social al servicio de la investigación. IJERI: International Journal of Educational Research and Innovation, (5), 170-183.

Rúas-Araújo, J., Fernández-Holgado, J. y Alén-Amil, J.Á. (2018). La regulación de los debates electorales en el servicio audiovisual público. Revista Ibérica de Sistemas e Tecnologías de Informaçao, (16), 158-170.

Rúas-Araújo, J., Puentes-Rivera, I. y Dapena-González, B. (2015). Neuropolítica, emoción y sentimiento en las redes sociales. En F. Campos-Freire y J. Rúas-Araújo (Eds.). Las redes sociales digitales en el ecosistema (pp. 53-71). Cuadernos Artesanos de Comunicación.

Rueda-Ortiz, R. (2015). Redes sociales digitales: de la presentación a la programación del yo. Iztapalapa. Revista de Ciencias Sociales y Humanidades, 36(78), 71-101.

Saavedra-Llamas, M., Rodríguez-Fernández, L. y Barón-Dulce, G. (2015). Audiencia social en España: Estrategias de éxito en la televisión nacional. Icono 14, (13), 215-237. http://doi.org/10.7195/ri14.v13i2.822

Sequera-Díaz, R. (2013). Televisión y Redes Sociales: nuevo paradigma en la promoción de contenidos televisivos. Ámbitos: Revista Internacional de Comunicación, (22), 1-16.

Serrano-Puche, J. (2012). Herramientas web para la medición de la influencia digital: análisis de Klout y PeerIndex. El profesional de la información, 21(3), 298-303. http://doi.org/10.3145/epi.2012.may.11

Soengas-Pérez, X. y Rodríguez-Vázquez, A. I. (2014). El control gubernamental de RTVE y el pluralismo en los informativos. Estudios sobre el Mensaje Periodístico, 21(2), 1225-1240. http://doi.org/10.5209/rev_ESMP.2015.v21.n2.50912

Soengas-Pérez, X., Elías-Pérez, C. y López-Cepeda, A. M. (2018). El tratamiento de la información política e institucional en TVE. Revista Latina de Comunicación Social, (73), 1072-1089. http://doi.org/10.4185/RLCS-2018-1297

Téllez, N., Muñiz, C. y Ramírez, J. (2010). Función discursiva en los debates televisados. Un estudio transcultural de los debates políticos en México, España y Estados Unidos. Palabra Clave, 13(2), 251-270. http://doi.org/10.5294/pacla.2010.13.2.2 
Tjong, E. \& Bos, J. (2012). Predicting The 2011 Dutchd Senate Election Results With Twitter. Association for Computational Linguistics. Proceedings of the 13tj Conference of the European. Avignon, Francia.

Toledo-Bastos, M. Galdini-Raimundo, R. \& Travitzki, R. (2013). Gatekeeping Twitter: message diffusion in political hashtags. Media, Culture \& Society, 35(2), 260-270. http://doi.org/10.1177/0163443712467594

Tumasjan, A., Sprenger, T., Sandner, P.H. \& Welpe, I. (2010). Predicting Elections with Twitter: What 140 Characters Reveal about Political Sentiment. Proceedings of the Fourth International Conference on Weblogs and Social Media, ICWSM 2010. Washington, DC, USA.

Vergeer, M. y Hans Franses, P. (2015). Live audience responses to live televised election debates: time series analysis of issue salience and party salience on audience behaviour. Information, Communication \& Society, 19(10), 1390-1410. http://doi.org/10.1080/1369118X.2015.1093526

\section{AUTORES:}

\section{Belén Galletero Campos}

Profesora de la Facultad de Comunicación de la Universidad de Castilla-La Mancha. Forma parte del equipo de investigación de dos proyectos nacionales de $\mathrm{I}+\mathrm{D}$ y ha dirigido proyectos de investigación con empresas y entidades como el Instituto de la Mujer de Castilla-La Mancha o la ONG Plan Internacional. Forma parte del grupo de investigación Mediacom, orientado a investigar sobre la estructura de la comunicación en la comunidad autonóma. Ha realizado estancias de investigación en el Centro de Estudos de Comunicação e Sociedade de la Universidade do Minho (PT) y ha colaborado en el proyecto Re/media.Lab - Laboratório e Incubadora de Media Regionais (Universidade da Beira Interior, PT). Es miembro del Consejo Asesor de Radiotelevisión Castilla-La Mancha en representación de la Universidad de Castilla-La Mancha.

belen.galletero@uclm.es

Î́ndice H: 2 (diciembre de 2019).

Orcid ID: https://orcid.org/0000-0002-9549-9507

Google Scholar: https://scholar.google.es/citations?user=pRNNxaQAAAAJ\&hl=es

\section{Ana María López Cepeda}

Licenciada en Periodismo por la Universidad de Santiago de Compostela (USC), Licenciada en Derecho por la Universidad Nacional de Educación a Distancia (UNED) y Doctora en Comunicación y Periodismo por la Universidad de Santiago de Compostela (USC). En la actualidad es Profesora en la Universidad de Castilla-La Mancha (UCLM) en donde imparte materias sobre Estructura de Medios, Sistema de Medios y Políticas Públicas de Comunicación. Es Investigadora Principal del Grupo de Investigación en Sociología de la Comunicación de la UCLM. Como investigadora, sus áreas de especialización son las políticas públicas de comunicación, políticas audiovisuales, derechos de autor y periodismo, estructura de grupos de comunicación y medios audiovisuales de servicio público. En el ámbito profesional, trabajó como periodista y redactora en prensa escrita y gabinetes de comunicación.

ana.lopezcepeda@uclm.es

Î́ndice H: 5 (diciembre de 2019).

Orcid ID: http://orcid.org/0000-0001-8328-9142

Google Scholar: https://scholar.google.es/citations?user=cJm7LFIAAAAJ\&hl=es 


\section{Arturo Martínez Rodrigo}

Arturo Martínez Rodrigo es profesor Ayudante Doctor en la Facultad de Comunicación de la Universidad de Castilla-La Mancha (UCLM). Es Ingeniero Técnico de Telecomunicaciones y Graduado en Sistemas Audiovisuales de Telecomunicación por la Universidad de Castilla-La Mancha (UCLM) y Doctor por la UCLM con Premio Extraordinario de Doctorado en el área de Ingeniería. Se especializó en el procesado y análisis de datos con especial énfasis en el diseño estadístico de modelos de aprendizaje automático y clasificadores avanzados. Ha realizado estancias de investigación en la University of Surrey $(U K)$, en el Departament of Mechanical Sciences, y en el Intelligent System Laborayory en la Universidade do Minho (PT). Es autor de más de 50 trabajos científicos en este campo y experto en el estudio de dinámica no lineal y su aplicación al mundo de los datos, tal y como puede derivarse de su investigación y proyectos estatales donde participa. arturo.martinez@uclm.es

Índice H: 11 (diciembre de 2019).

Orcid ID: https://orcid.org/0000-0003-2343-3186

Google Scholar: https://scholar.google.es/citations?user=mJ1GX94AAAAJ\&hl=es\&oi=ao 